\title{
The Creation of a Strong Magnetic Field by Means of Large Magnetic Blocks from NdFeB Magnets in Opposing Linear Halbach Arrays
}

\author{
Václav Žežulka and Pavel Straka* \\ Institute of Rock Structure and Mechanics, Academy of Sciences of the Czech Republic, v.v.i. \\ V Holešovičkách 41, 18209 Prague 8, Czech Republic
}

(Received 12 February 2016, Received in final form 19 July 2016, Accepted 20 July 2016)

\begin{abstract}
The article presents the results and findings obtained through the assembly of opposing linear Halbach arrays from two magnet layers using large magnetic blocks from permanent $\mathrm{NdFeB}$ magnets, especially concerning the distribution of magnetic induction in an air gap. The use of these large blocks has led to a significant expansion of the area of magnetic field with a substantially higher value of magnetic induction in comparison with similar linear Halbach arrays composed of small magnets. The paper also discusses the determined dependences of magnetic induction on the parameters of the $x, y, z$ coordinate system and indicates the possibilities of achieving an even stronger magnetic field in a larger volume of an air gap for application for instance in equipment for magnetic separation of raw materials, in instrument technologies and in other areas.
\end{abstract}

Keywords : magnetic field, permanent magnets, $\mathrm{NdFeB}$ magnets, Halbach arrays

\section{Introduction}

At present, the equipment for mineral processing and instrument technology is becoming increasingly attractive for use. Many of these applications require the use of magnetic fields with high values of magnetic induction or with an extreme gradient of this parameter. Thanks to high values of remanent magnetization and flux density, $\mathrm{NdFeB}$ permanent magnets are predetermined for these purposes. The work [1] describes a geometric arrangement of permanent $\mathrm{NdFeB}$ magnets for magnetic resonance: In the implemented large device with $\mathrm{NdFeB}$ magnets from the material N42, it generates, in an air gap of $0.06 \mathrm{~m}$ and a pole diameter of $0.2 \mathrm{~m}$, a magnetic field of an induction of 1.6 T. Simultaneously, the authors mention that the use of the Halbach ring structure would make it possible to attain high fields and reduce the weight of the device, but this structure is too complicated to be manufactured and assembled.

With simplified Halbach cylinders, values of up to 1.8 $\mathrm{T}$ were attained [2]. In the case of Halbach cylinders with iron-pole magnetic-flux concentrators, the maximum level shifted to a value of $3.9 \mathrm{~T}$, as the work [3] proves. Using

(C)The Korean Magnetics Society. All rights reserved.

*Corresponding author: Tel: +420-266-009-402

Fax: +420-284-680-105, e-mail: straka@irsm.cas.cz an original source of a magnetic field on the basis of permanent magnets in a Halbach sphere, the calculated value of magnetic induction in the central area $(\phi 6 \mathrm{~mm} \times$ $h 2.8 \mathrm{~mm}$ ) exceeded the level of $4.3 \mathrm{~T}$ [2]. Besides the value of the field generated, in many cases it is important to monitor the uniformity of this field as well. A solution to this problem has been discussed on the basis of Halbach geometrical configurations in the cases of a ring dipole, a square dipole and a C-dipole [3].

At lower field values (below $0.4 \mathrm{Br}$ ), the constructions of a C-dipole type are the most advantageous, whereas square and ring dipoles have been shown to be the best in the resolution of fields with high values of magnetic flux. In systems with magnetic cooling, it is necessary to create some areas with a high and others with a low value of magnetic flux. The application of the Halbach cylinder, where some of the permanent magnets have been replaced by a magnetically soft material with a high value of relative permeability, can make it possible to achieve an almost 50\% increase in the difference between the magnetic-induction values in the areas with high and low magnetic field levels [4]. Other publications, e.g. [5], have described constructions of autonomous sources of a strong magnetic field for magnetic separation chiefly in ceramic technological processes or equipment usable for different purposes [6, 7].

A great deal of attention has been devoted to simulations 
of strong magnetic fields. Hilton and McMurry [8] have examined and idealized a model composed of infinite line dipoles and performed computational simulations on a realizable device using a magnetic boundary element method. Furthermore, optimization methods have been presented in the design of Halbach arrays to maximize the forces applied to magnetic nanoparticles at deep tissue locations [9]. The methods presented, based on semidefinite quadratic programming, have yielded provably globally optimal Halbach designs in 2D and 3D for the maximum pull or push magnetic forces. Choi and Yoo [10] have designed a Halbach magnet array using a numerical optimization method based on finite-element analysis. The optimal magnet arrays composed of two and three linear magnet layers have been investigated to increase the attractive, repulsive and tangential magnetic forces between magnet layers. In the work [11], permanent magnet designs in which the magnetic flux density can be altered are analyzed using numerical simulations and compared based on the generated magnetic flux density in a sample volume and the amount of the magnet material used.

Marble claims [12] that through a relatively simple arrangement of permanent magnets it is feasible to achieve more than $80 \%$ of the maximum possible magnetic field. On this basis, the work [13] describes the use of a geometrical configuration of permanent magnets consisting of opposing geometrically linear assemblies based on Halbach arrays for the generation of strong magnetic fields, which have been theoretically modeled and then experimentally verified. The implementation of these opposing assemblies using $\mathrm{NdFeB}$ magnets provided a significantly higher value of magnetic induction in the middle of an air gap in comparison with the classic magnet arrangement. Simultaneously, however, unlike in the classic parallel configuration, opposing Halbach assemblies have shown a significant decrease of magnetic induction values when passing from the middle of the assemblies in the direction parallel to the magnet surfaces.

From this brief summary, it can be deduced that: (a) the linear Halbach array as a structurally simple construction is applicable to the creation of a transportable device; (b) under certain conditions, it is possible to achieve a higher magnetic field in the air gap between two linear Halbach arrays.

The objectives of this work are to present the design of opposing linear Halbach assemblies from large NdFeB magnet blocks, which provides a higher magnetic field (in comparison with the two opposing magnet blocks shown in Fig. 6) in a relatively large volume, and to describe the distribution of magnetic field intensity in the specific case realized. The different types of arrays assembled from individual magnets by means of a small experimental device have already been compared earlier [13].

\section{Theory}

As mentioned, the main advantage of the linear Halbach array is that it is easy to construct using simple rectangular magnet blocks. As a theoretical basis for this construction, the course of magnetic induction in the air gap between two opposing Halbach arrays (two magnet layers [10]) was computed. The model of the setup can be formulated using a scalar magnetic potential. Two types of sub-regions can be distinguished: source-free medium (air) and magnetic material (permanent magnets). Generally, magnetic induction is defined as:

$$
\vec{B}=\mu_{0} \vec{M}+\mu_{0} \vec{H}
$$

where $\vec{M}$ is the magnetization and $\vec{H}$ is the magnetic field intensity. In our case, it is assumed that the magnetization in the permanent magnet is homogeneous and the medium is magnetically isotropic. The magnetic induction must satisfy the condition:

$$
\nabla \cdot \vec{B}=0 \text {. }
$$

In order to compute the distribution of the magnetic field, the field will be expressed using a scalar magnetic potential defined as:

$$
\vec{H}=-\nabla \Phi \text {. }
$$

The insertion of this expression for the scalar magnetic potential in (1) and combining the result with the divergence condition (2) leads to the following [14]:

$$
\nabla \cdot \mu_{0} \nabla \Phi=\nabla \cdot \mu_{0} \vec{M}
$$

Equation (4) constitutes the formulation governing the scalar magnetic potential solution. Both types of the mentioned sub-regions are considered without free currents; the result is that the tangential components of the magnetic field intensity along their mutual boundaries are continuous. Equation (4) can be treated as governing the differential equation on the domain:

$$
L \Phi=f,
$$

where $L$ is the differential operator and $f$ is the so-called excitation or forcing function; together with the appropriate boundary condition, the latter defines a boundaryvalue problem for the scalar magnetic potential F. A solution to this problem can be obtained using the finite element method (FEM). The subsequent computational procedure can be found in the work [14]. On this basis, 
the course of the values of magnetic induction in the gap between two opposing Halbach arrays was computed and the configuration of small $\mathrm{NdFeB}$ permanent magnets consisting of opposing geometrically linear assemblies based on Halbach arrays was implemented as mentioned above.

Both the computer simulation and the implementation have confirmed that using these opposing linear assemblies with $\mathrm{NdFeB}$ magnets with a high energy product, it is possible to achieve (in the defined volume) magnetic fields with magnetic flux density values exceeding the level of the remanent magnetization of the permanent magnets used. The application of large magnetic blocks from the same material thus opens a real possibility of the creation of a strong magnetic field in a greater volume for various applications.

\section{Experiments}

\subsection{Opposing linear Halbach arrays with large blocks from $\mathrm{NdFeB}$ magnets}

For the creation of a magnetic field in an air gap between linear assemblies of permanent magnets, two opposing Halbach arrays of the same size have been selected with respect to the results obtained earlier [13]. They must be placed in such a configuration that the one-sided magnetic fields of both assemblies act in the area of the air gap while respecting the direction of the magnetic field. This arrangement is depicted in front and side views in Fig. 1. Each assembly comprises two large side blocks

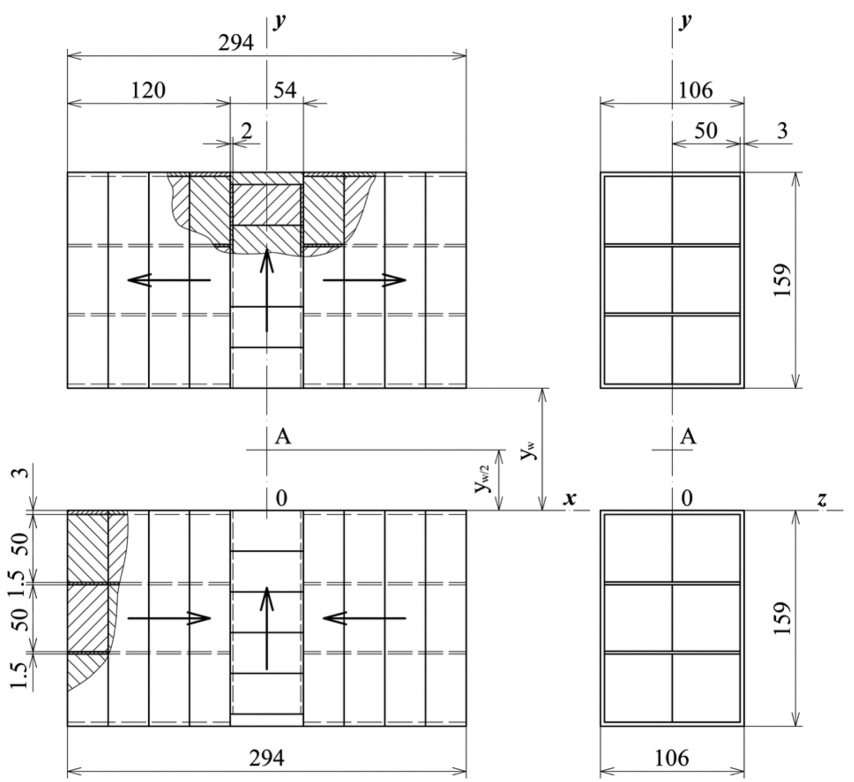

Fig. 1. Opposing linear Halbach arrays with large blocks from NdFeB magnets.

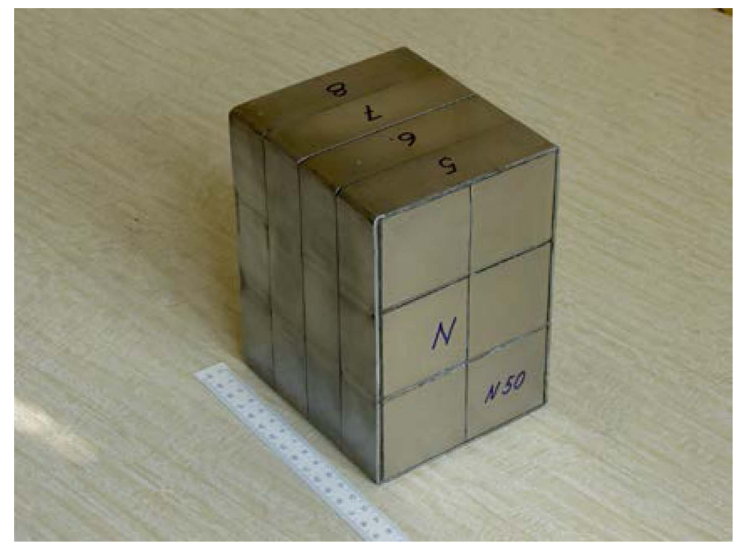

Fig. 2. (Color online) A side magnetic block.

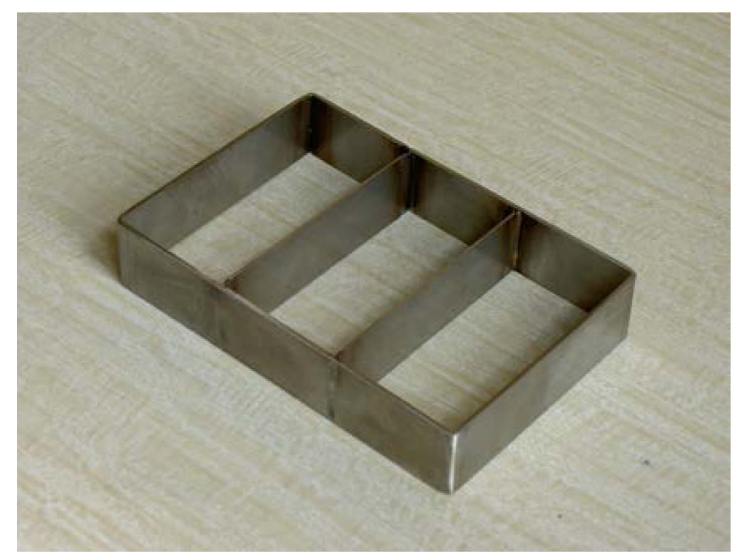

Fig. 3. (Color online) A peripheral frame with internal partitions.

and one central block. For a better idea of the internal arrangement of the blocks, Fig. 1 shows their partial imaginary cross-sections (hatched areas).

Each large side block (Fig. 2) was assembled from four compact magnetic plates. The individual magnetic plates of a height of $0.03 \mathrm{~m}$ were always composed of six pieces of $\mathrm{NdFeB}$ magnets with the dimensions $0.05 \times 0.05 \times$ $0.03 \mathrm{~m}$, placed in a peripheral non-magnetic steel frame (with two internal partitions) (Fig. 3). These were sealed with epoxy resin. After the epoxy resin was cured, each plate was magnetized as a whole. All the plates were magnetized in the same direction.

The method used for the assembly of magnetized compact plates into blocks [15] makes it possible to control the speed of the attraction of the magnets during their assembly in the direction perpendicular to the future common contact surface. In this case, the magnetic plates are gradually inserted into the preparation tube, filled with a liquid (e.g. hydraulic oil), with the adjacent surfaces of the magnets having the opposite polarity. Through a controlled discharge of the liquid from the space between 
the magnetic plates, one can control the speed of their attraction to prevent their strong and thus very rapid attraction and subsequent damage. This method also allows the elimination of the possible partial demagnetization of the magnets (or magnetic plates) during their assembly [5]. The overall dimensions of the side block of a total weight of approximately $15 \mathrm{~kg}$ were $0.159 \times 0.106$ $\times 0.120 \mathrm{~m}$. The ground-plan dimensions of the side block, $0.159 \times 0.106 \mathrm{~m}$, are thus identical with the ground-plan dimensions of each compact magnetic plate and are determined by the use of the already-mentioned six pieces of magnets placed in a peripheral frame with some clearance to be filled with epoxide resin. In addition, these ground-plan dimensions must correspond to the internal dimensions of the tube, through which individual magnetic plates go with little clearance when the block is being assembled. The height of the block is determined by the number of these assembled plates and has been limited to four plates of the total height of $120 \mathrm{~mm}$, because a further increase in the height of the block at the given constant ground-plan dimensions brings only a small increase in the magnetic induction in the air gap ([5], Fig. 10) and has practically no significance. Other limitations concerning possible bigger dimensions (both the height and the ground plan) have arisen from its growing weight. The currently used side block of a weight of $15 \mathrm{~kg}$ can be carefully handled manually without various auxiliary handling devices, which would, however, be necessary if the weight of the block were further increased.

The central magnetic block (Fig. 4) has been assembled similarly from five smaller magnetic plates, each of which was again $0.03 \mathrm{~m}$ high. In this case, the compact magnetic plate consists of magnets of the same size as in the previous case, likewise placed in a peripheral non-magnetic steel frame, this time without internal partitions. The overall size of the central block was $0.054 \times 0.106 \times$

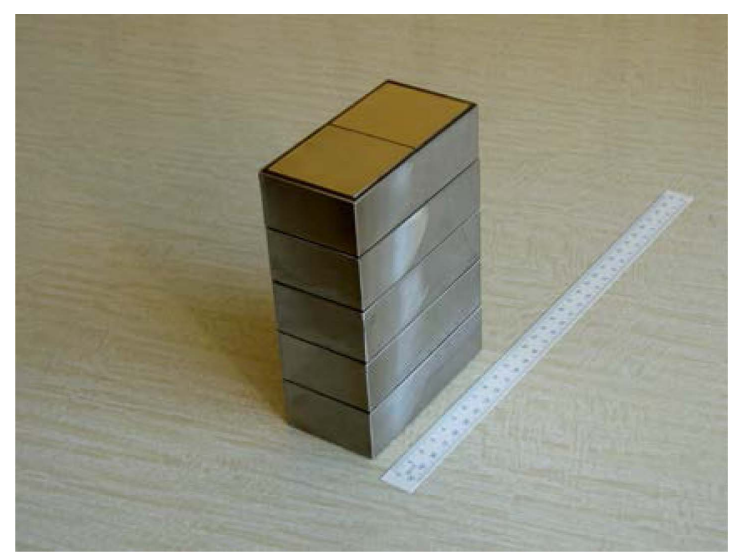

Fig. 4. (Color online) A central magnetic block.
$0.150 \mathrm{~m}$ and its weight was $6.35 \mathrm{~kg}$. As suitable low magnets that would make it possible to reach the height of $0.159 \mathrm{~m}$ like in the side blocks were not available, nonmagnetic shims were placed under the central blocks to raise them up to this height. Of course, this solution has some negative yet not substantial impact on the attained values of magnetic induction. The width of the central block, $0.054 \mathrm{~m}$, was set with respect to the overall dimensions of the assembly of the magnet blocks so as to preserve approximately the same proportions, evident from the figures and text in [12], where Marble mentions the optimization of the width of the central magnet in the Halbach array in order to obtain the strongest possible magnetic field at a remote point.

In this connection, it is also necessary to mention the reasons for the use of peripheral non-magnetic frames during the assembly of compact magnetic plates. Firstly, after the whole plate has been magnetized, these stainless frames capture the mutual repulsive forces between the individual magnets and thus prevent the disintegration of the plate. Secondly, the frames protect these plates from damage and possible destruction during their assembly into magnetic blocks and during manipulation with these blocks. The use of the frames hence increases the work safety mainly in the cases of magnets and magnetic blocks of large size. In the case of the above-mentioned central blocks, however, the peripheral frames had to be used because of the size of the magnets available during the assembly. After each Halbach array has been assembled, the walls of these frames form basically two air gaps of a width of $2 \mathrm{~mm}$ (see Fig. 1), which undoubtedly reduce the values of magnetic induction attainable in the air gap between the arrays. If a magnet of $0.1 \times 0.05 \times 0.03 \mathrm{~m}$ is utilized to replace two magnets of half the length used in the plate and it is handled carefully, one can create a central block without peripheral frames and thus, by removing the mentioned air gaps, increase the magneticinduction values in the gap.

After the assembly, the total weight of both opposing linear Halbach arrays was approximately $73 \mathrm{~kg}$, out of which the net weight of $\mathrm{NdFeB}$ magnets was $65.3 \mathrm{~kg}$. The two assemblies comprised a total of 116 magnets of the same size $(0.05 \times 0.05 \times 0.03 \mathrm{~m})$ from the material N50 with these parameters: remanence $B_{r}=1.415 \mathrm{~T}$, coercivity $H_{c b}=1065 \mathrm{kA} / \mathrm{m}, H_{c j}=1098 \mathrm{kA} / \mathrm{m}$ and maximum energy product $(B H)_{\max }=385 \mathrm{~kJ} / \mathrm{m}^{3}$.

It is evident that the opposing assemblies from magnetic blocks depicted in Fig. 1 are the target of both the individual assemblies and their opposing arrangement. Because of large mutual magnetic forces, however, such assemblies cannot be created and further maintained in 


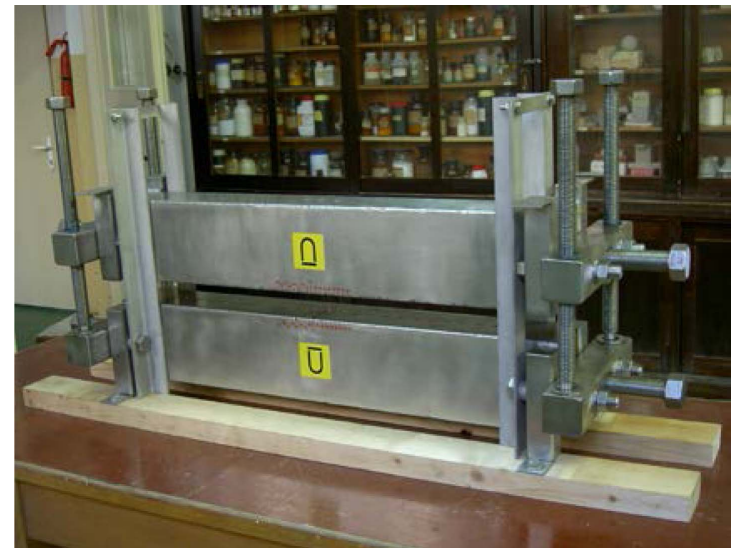

Fig. 5. (Color online) The device for the creation of the opposing assemblies from large magnetic blocks.

the desired opposing position without an auxiliary device (preparation). A device enabling this is shown in Fig. 5. It consists of two opposing hollow tubes made from nonmagnetic steel with the inner dimensions corresponding to the size of each array. Their distance (the width of the air gap) may be continuously set in the range of 0.3-0.01 $\mathrm{m}$. Each tube has removable parts at its ends, enabling a gradual insertion of magnetic blocks into the tube and their subsequent pressing together into the final assembly. Each completed assembly may be simply moved to set the two assemblies exactly opposite each other.

Before the creation of the opposing assemblies began, the entire device was thus completely dismantled and both arrays were assembled in individual, separated tubes. Afterward, the upper part of the device with a preset maximum distance between the assemblies was set to a specific position with respect to the bottom tube. Step by step, the upper assembly was moved closer to the lower one.

For the sake of completeness, one should mention that, should the need arise, the selected solution allows for smooth dismantling of the arrays in reverse order down to individual large blocks and then to individual compact plates. If necessary, these plates can be magnetized again and used in the same or other device.

\subsection{Two opposing large magnetic blocks from NdFeB magnets}

This simplest possible design of opposing magnets has been selected for comparison with the previous case. It is again depicted in front and side views including dimensions in Fig. 6. In this case, the same magnetic blocks as in Fig. 2 were turned with their poles opposite one another, placed in the opposing tubes of the device in Fig. 4 and set precisely opposite each other.
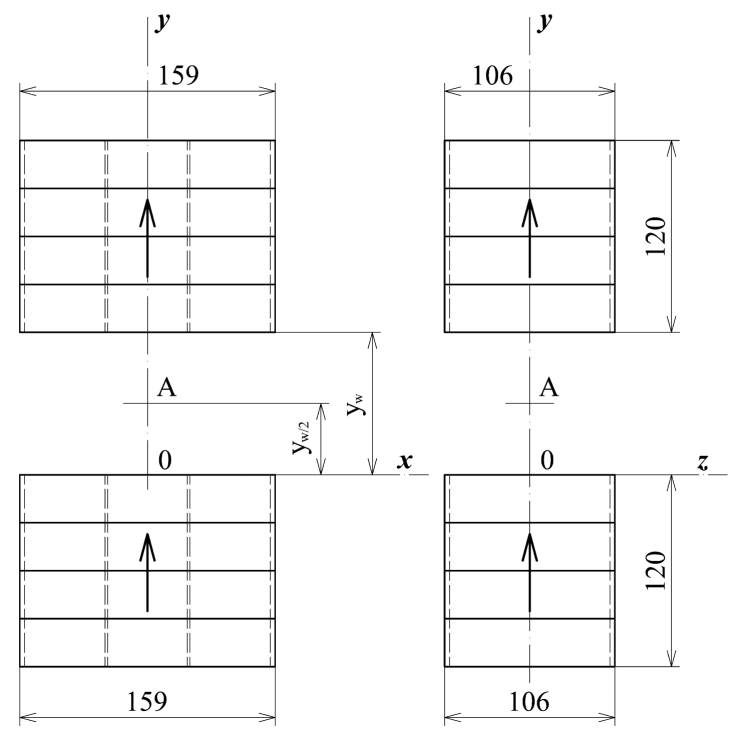

Fig. 6. Two opposing large magnetic blocks from $\mathrm{NdFeB}$ magnets.

\subsection{Measurement method}

For the measurements of all dependences, the beginning of the coordinates $x, y$ and $z$ was selected in the middle of the surface of the lower array (point 0 in Fig. 1 and Fig. 6). In order for the values attained and the course of magnetic induction to be comparable with results obtained earlier, the measurement methodology used was similar to that utilized in previous work [13]. The measurements of the magnetic induction in the individual configurations were performed using the same F.W. Bell Teslameter, type 5080, with the Hall transverse probe.

When the dependence $B_{y w / 2}=f\left(y_{w}\right)$ was measured, the upper array of magnets (the upper tube) was gradually, step by step, moved closer to the solid bottom array in the bottom tube. For the selected setting of the width of the air gap $y_{w}$, magnetic induction was measured in the middle of the width of this gap, always using a solid non-magnetic spacer support/pad under a probe of a height equal to $y_{w / 2}$ minus $1 / 2$ the thickness of the probe for its more precise setting.

For the entire measurement of the dependence $B_{y w 10}=$ $f(x)$, the width of the air gap was set to be constant. Magnetic induction was measured in the middle of this air gap at individual points along the $x$-axis to both left and right of point 0 based on the scale marked on the bottom as well as upper tubes (see Fig. 5), again with the use of a spacer support/pad below the probe.

The measurement of the dependence $B_{y w 10}=f(z)$ was carried out similarly to the previous case. Magnetic induction was again measured in the middle of the air gap at individual points along the $z$-axis to the left and right of 
point 0 based on another drawn scale. The probe was placed on a solid spacer support/pad again.

\section{Results and Discussion}

4.1. The measurements of the dependences of magnetic induction in the case of linear opposing Halbach arrays with large blocks from $\mathrm{NdFeB}$ magnets

The dependences of magnetic induction in the case of opposing linear Halbach arrays composed of small $\mathrm{NdFeB}$ magnets from the material N45 are depicted in the following respective Figs. 7, 8 and 9. In this case, the magnetic properties of the material $\mathrm{N} 45$ ([13], p. 8) are similar to the material N50 mentioned here.

4.1.1. The dependence of magnetic induction in the middle of an air gap $B_{y w / 2}$ on the width of this gap $y_{w}$ with $y_{w}$ ranging from $300 \mathrm{~mm}$ to the shortest possible distance between the assemblies in the preparation equal to $10 \mathrm{~mm}$. This dependence is depicted in Fig. 7 (red curve, triangles) along with the corresponding dependence determined earlier [13] (blue curve, squares).

4.1.2. The dependence of magnetic induction $B_{y w 10}$ in the middle of an air gap of the constant width $y_{w}=20$

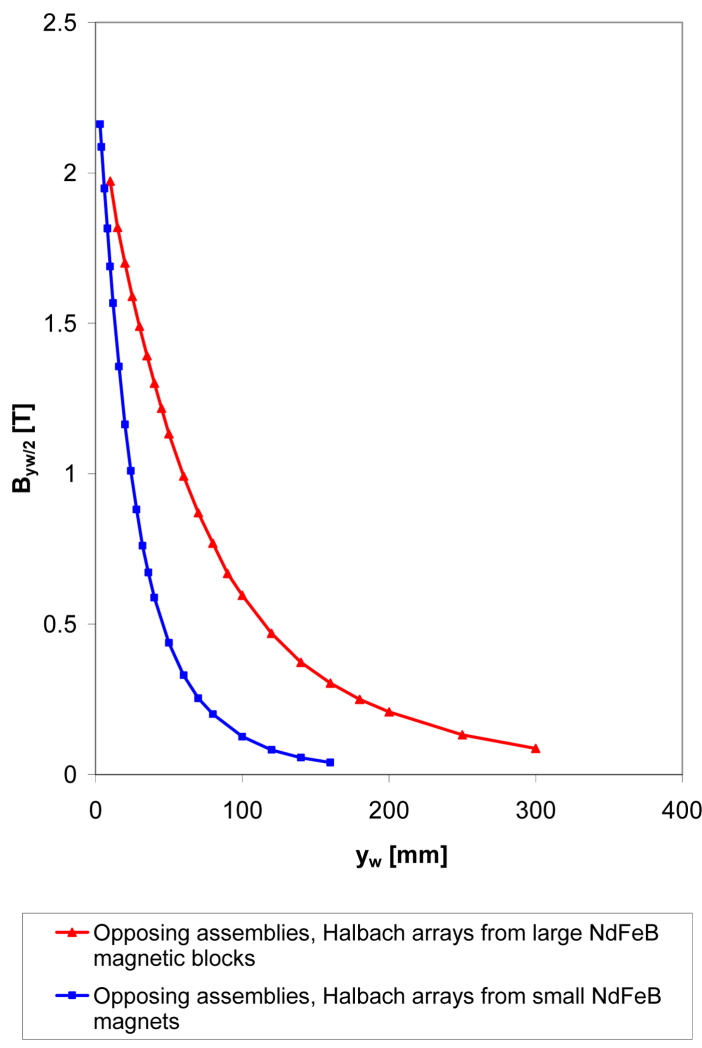

Fig. 7. (Color online) Dependence $B_{y w / 2}=f\left(y_{w}\right)$.

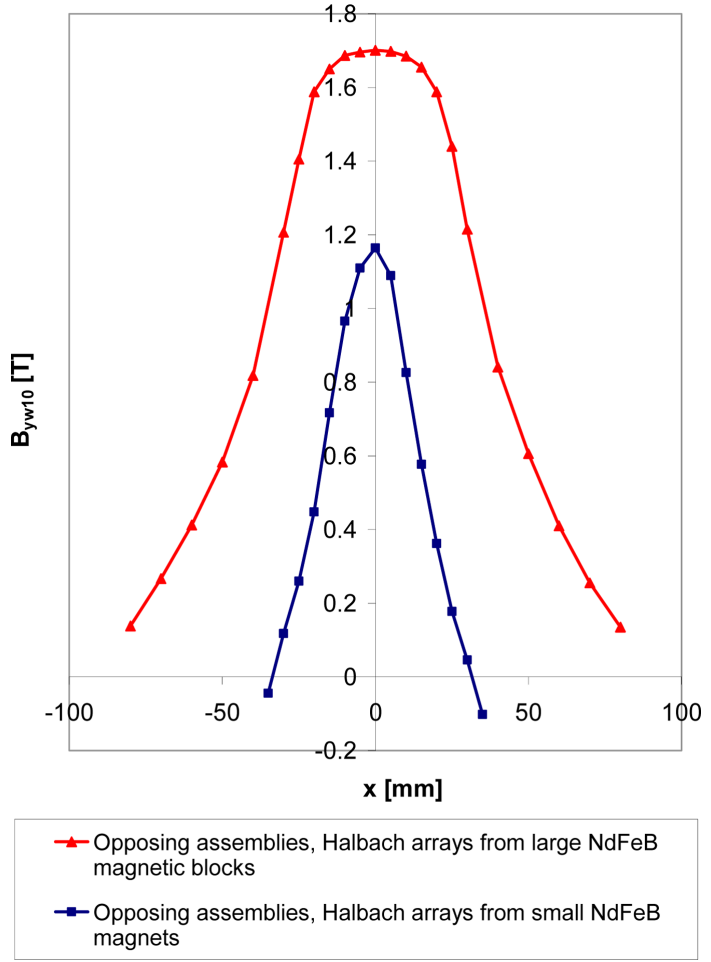

Fig. 8. (Color online) Dependence $B_{y w 10}=f(x)$ for $y_{w}=$ const. $=20 \mathrm{~mm}$.

$\mathrm{mm}$ (at the level of $y_{w / 2}=$ const. $=10 \mathrm{~mm}$ ) on the parameter $x$ (the $x$-axis is parallel to the surface of the magnets) with $x$ ranging from $-80 \mathrm{~mm}$ to $+80 \mathrm{~mm}$. The measured dependence is depicted in Fig. 8 (red curve, triangles), for comparison again with the corresponding dependence determined earlier.

4.1.3. The dependence of magnetic induction $B_{y w 10}$ in the middle of an air gap of the constant width $y_{w}=20$ $\mathrm{mm}$ (at the level of $y_{w / 2}=$ const. $=10 \mathrm{~mm}$ ) on the parameter $z$ (the $z$-axis is perpendicular to the $x$-axis) with $z$ ranging from -50 to $+50 \mathrm{~mm}$. The measured dependence is depicted in Fig. 9 (red curve, triangle), along with the corresponding dependence measured in the case of linear Halbach arrays from small blocks.

4.1.4. Discussion - Opposing linear Halbach arrays with large magnetic $\mathrm{NdFeB}$ blocks. Figures 7, 8 and 9 indicate that, in comparison with assemblies from small magnets, substantially higher values of magnetic induction are attained in a larger volume of the air gap in assemblies from large magnetic blocks.

It arises from Fig. 7, for instance, that the magnetic induction achieved in assemblies from large magnetic blocks in the same air gap of a width of $140 \mathrm{~mm}$ is approximately 6.66 times higher. The smaller the width of 


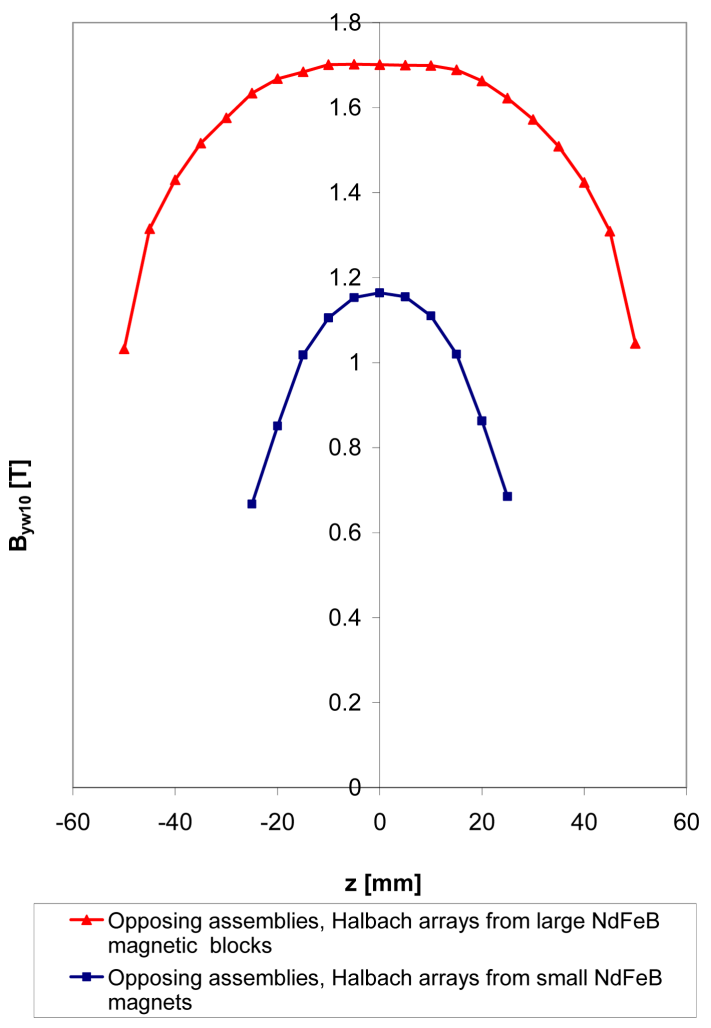

Fig. 9. (Color online) Dependence $B_{y w 10}=f(z)$ for $y_{w}=$ const. $=20 \mathrm{~mm}$.

the air gap, the lower the increase; for example in the case of the same gap of a width of $60 \mathrm{~mm}$, this magnetic induction is 3 times higher; in a 20 -mm width, it is still 1.46 times higher. On the other hand, a comparison of the same values of magnetic induction achieved indicates that e.g. an approximately the same induction of $0.59 \mathrm{~T}$ was achieved in large-block assemblies in a 100-mm gap as against a 40-mm gap in small-magnet assemblies. The increase in the width of the air gap is hence 2.5 times higher.

Figure 8, depicting $B_{y w 10}$ values (in the middle of an air gap of the constant width of $20 \mathrm{~mm}$ ) in dependence on the parameter $x$ clearly shows not only the above-mentioned 1.46 times higher magnetic induction achieved in Point A, but especially the substantially greater width of the area of the strong field.

It thus arises from a comparison of the measured values in Figs. 7, 8 and 9 that the use of large magnetic $\mathrm{NdFeB}$ blocks of the mentioned dimensions and material in linear opposing Halbach arrays has made it possible to achieve in an air gap of a width of $20 \mathrm{~mm}$ a magnetic field in a roughly 17 times higher air-gap volume of and with the value of magnetic induction 1.5 times higher than in the field attained with similar assemblies from small $\mathrm{NdFeB}$ magnets.
4.2. The measurements of the dependences of magnetic induction in the case of two opposing large magnetic blocks from $\mathrm{NdFeB}$ magnets

The dependences were measured like in the case of the opposing Halbach arrays; for comparison, Figures 10 and 11 show again the dependences determined in the case of opposing small $\mathrm{NdFeB}$ magnets in this simplest arrangement (blue curves, squares).

4.2.1. The dependence $B_{y w / 2}$ on the width of the air gap $y_{w}$ is depicted in Fig. 10 (red curve, triangles), which includes, besides the above-mentioned blue curve, also the dependence determined earlier ([5], Fig. 9), pertaining to two opposing blocks of the same size placed in a closed iron circuit (violet curve, diamonds).

4.2.2. The dependence $B_{y w / 2}$ on the parameter $x$ is depicted in Fig. 11, showing, besides the blue curve, pertaining to small magnets, also the dependences $B_{y w / 2}$ in the middles of air gaps of $20 \mathrm{~mm}, 50 \mathrm{~mm}$ and $80 \mathrm{~mm}$ (at constant levels $y_{w / 2}$ equal to $10 \mathrm{~mm}, 25 \mathrm{~mm}$ and 40 $\mathrm{mm})$.

4.2.3. The dependence $B_{y w / 2}$ on the parameter $z$ is depicted in Fig. 12, again in the middles of air gaps of widths of $20 \mathrm{~mm}, 50 \mathrm{~mm}$ and $80 \mathrm{~mm}$, i.e. at constant
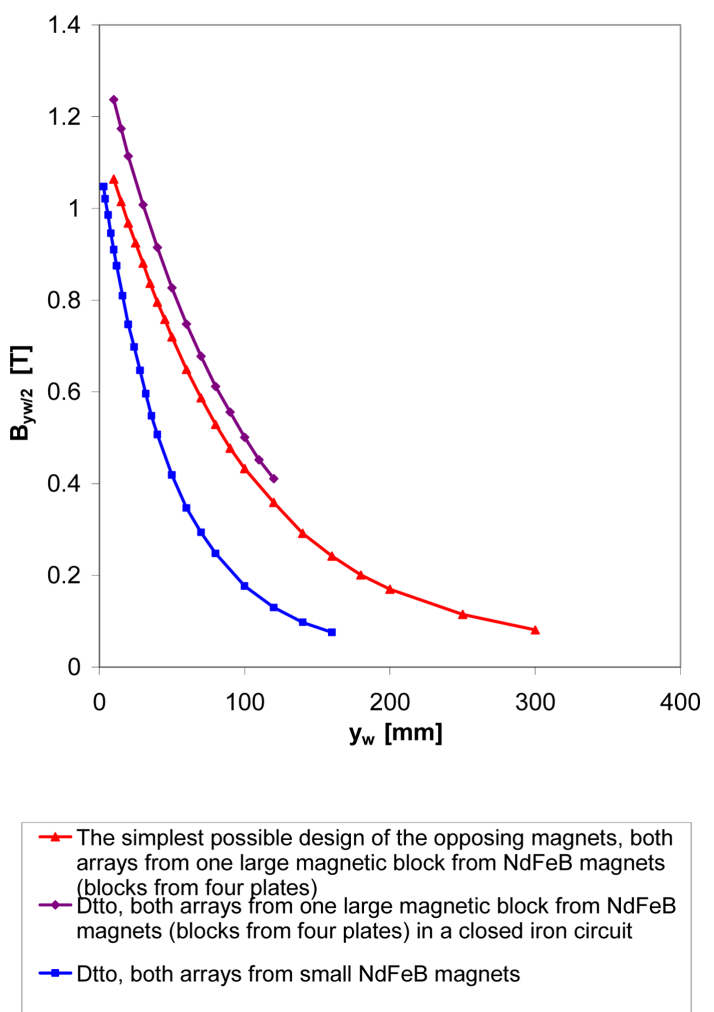

Fig. 10. (Color online) Dependence $B_{y w / 2}=f\left(y_{w}\right)$. 


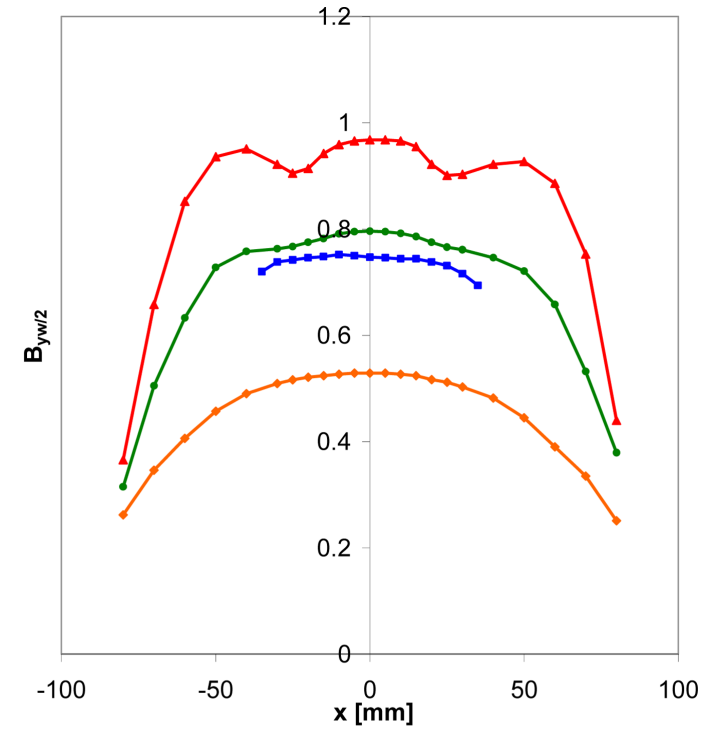

$\downarrow$ The simplest possible design of the opposing magnets, both arrays from one large magnetic NdFeB block, magnetic induction in the middle of the air gap of a width of $20 \mathrm{~mm}$

$\rightarrow$ Dtto, both arrays from one large magnetic NdFeB block, magnetic induction in the middle of the air gap of a width of $50 \mathrm{~mm}$

$\rightarrow$ Dtto, both arrays from one large magnetic NdFeB block, magnetic induction in the middle of the air gap of a width of $80 \mathrm{~mm}$

$\rightarrow$ Dtto, both arrays from small NdFeB magnets, magnetic induction in the middle of the air gap of a width of $20 \mathrm{~mm}$

Fig. 11. (Color online) Dependence $B_{y w / 2}=f(x)$.

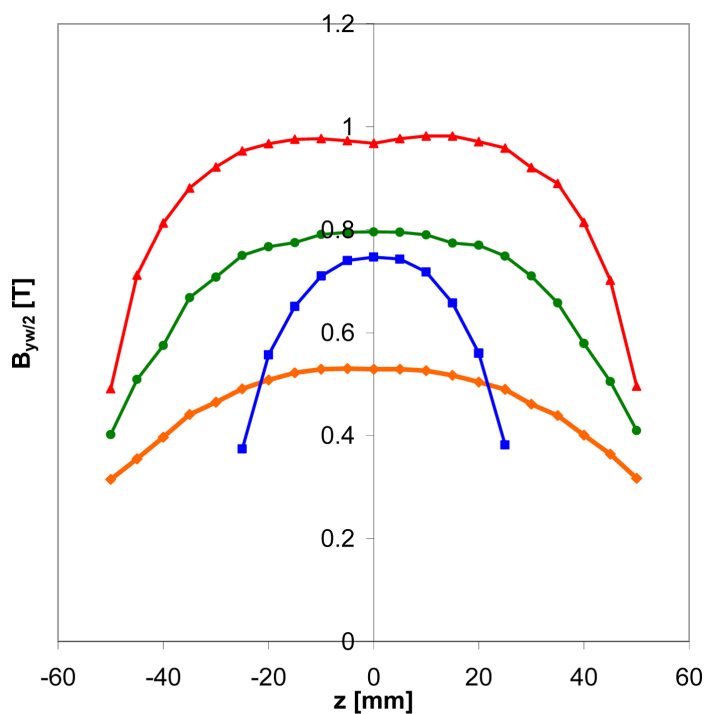

- The simplest possible design of the opposing magnets, both arrays from one large magnetic $\mathrm{NdFeB}$ block, magnetic induction in the middle of the air gap of a width of $20 \mathrm{~mm}$

$\rightarrow$ Dtto, both arrays from one large magnetic NdFeB block, magnetic induction in the middle of the air gap of a width of $50 \mathrm{~mm}$

$\rightarrow$ Dtto, both arrays from one large magnetic NdFeB block magnetic induction in the middle of the air gap of a width of $80 \mathrm{~mm}$

- Dtto, both arrays from the small NdFeB magnets, magnetic induction in the middle of the air gap of a width of $20 \mathrm{~mm}$

Fig. 12. (Color online) Dependence $B_{y w / 2}=f(z)$. levels $y_{w / 2}$ equal to $10 \mathrm{~mm}, 25 \mathrm{~mm}$ and $40 \mathrm{~mm}$.

4.2.4. Discussion-Opposing separate large magnetic blocks. Figures 10, 11 and 12 confirm the expectation that higher magnetic induction in a larger volume of the air gap is achieved even in this case of opposing large magnetic blocks than in opposing small magnets. In comparison with Halbach arrays from large blocks, however, the obtained values of magnetic induction are significantly lower, but in a larger volume of an air gap.

Even in this case (like in Halbach arrays), it is obvious from Fig. 10 that the magnetic induction achieved in a 140-mm-wide air gap is approximately three times higher in large opposing blocks than in the case of small opposing magnets. The lower the width of the air gap, the smaller the increase again; in a 20-mm gap, for instance, it is already only 1.3 times higher. A comparison of the same obtained values of magnetic induction shows that, for instance, approximately the same induction of $0.29 \mathrm{~T}$ was achieved in a 140-mm-wide gap in the case of opposing large blocks and in a 70-mm-wide gap in the case of opposing small magnets. The increase in the width of the air gap is thus twofold.

Figure 10 makes it possible to compare opposing large magnetic blocks placed in the mentioned stainless tubes (hence without an iron circuit; the red curve, triangle) with similar blocks of the same dimensions but placed in a closed iron circuit (the violet curve, diamonds). The dependences in this figure clearly indicate the amplifying effect of the closed magnetic circuit on the magnetic induction achieved in the air gap.

Figure 11, depicting the dependence $B_{y w / 2}$ in the middles of air gaps of various constant widths on the parameter $x$, shows in the case of the dependence corresponding to a constant width of $20 \mathrm{~mm}$ the significantly negative impact of the internal stainless partitions of the peripheral frames, which becomes gradually lower with the growing width. It needs to be mentioned in connection with these internal partitions that their use was necessitated by the reasons stated above, that is the dimensions of the magnets available. Currently, NdFeB magnets of substantially larger sizes are already commercially available, so that the compact magnetic plate of the overall size of the magnets 150 $\times 100 \times 30 \mathrm{~mm}$ could be made from only one piece of magnet with this overall dimension instead of the mentioned six pieces of small magnets. This would eliminate the use of internal partitions and with that also their mentioned negative impact. For further comparison, it is hence possible to ignore this influence and use the dependence $B_{y w / 2}=f(x)$ for a constant width of $20 \mathrm{~mm}$ in the range of the values $x$ from -50 and $+50 \mathrm{~mm}$ as uniform 
without the mentioned distortion.

Likewise in Fig. 12 in the dependence corresponding to the constant gap width of $20 \mathrm{~mm}$, a certain less significant distortion is evident in the point $z=0$. It is caused by a glued joint of two smaller magnets. With the growing width of the air gap, it disappears again. Like in the previous case, also here this distortion would entirely disappear if one large magnet were used instead of six small ones.

It hence arises from the evaluation of Figs. 10, 11 and 12 that the magnetic field in a 20-mm-wide air gap between the opposing separate large magnetic $\mathrm{NdFeB}$ blocks of the mentioned dimensions and material was achieved in an air-gap volume that was roughly 3.75 times larger and had the value of magnetic induction 1.3 times higher than the magnetic field obtained in the case of the opposing small NdFeB magnets.

An overall comparison of the opposing arrays and magnets implemented indicates that the opposing linear Halbach arrays make it possible to achieve much higher values of magnetic induction in the same air-gap width yet in a smaller air-gap volume when compared to two opposing magnets in the simplest arrangement (for example, the induction in the middle of a $20-\mathrm{mm}$-wide air gap is 1.56 times higher when small magnets are used and 1.76 times higher in the case of large blocks). Nevertheless, the results also reveal that the use of large blocks from $\mathrm{NdFeB}$ magnets in opposing linear Halbach arrays makes it possible to achieve a roughly seventeen-fold expansion of the area of the strong field with a 1.5 times higher value of magnetic induction in comparison with similar Halbach arrays assembled from small magnets.

\section{Conclusions}

The results obtained on the device implemented have confirmed that its principle might be, in the future, a viable path toward the creation of strong magnetic fields with no electrical energy consumption in various areas. This solution may be applied for instance in equipment in instrument technologies, in devices for magnetic separation of raw materials (which would be advantageous in the case of insufficient electric power input at the site of their treatment), in magnetic filters in the purification of diverse suspensions (for instance in the production of ceramics and porcelain, in food industry etc.), and quite likely in other areas as well. Nevertheless, a prerequisite for its application in any device is the possibility to create a strong magnetic field in a sufficiently large air-gap volume and width, necessary to fulfill the required function and parameters of the device concerned. A realistic way to meet this condition is the proposed use of large magnetic blocks consisting of strong $\mathrm{NdFeB}$ magnets placed in opposing arrays in tubes of corresponding sizes in the same way as small arrays from individual magnets.

It must be emphasized in this context that the transition from a small experimental device to a principally similar device of a large size is far from simple and requires addressing a number of problems that in small devices were either entirely absent or were only demonstrated to a negligible degree. These are issues related for instance to the method of the creation of magnets of large sizes, to the method of handling them safely, to the increase in their weight and mutual magnetic forces, which reach high values, and to the determination of the precise procedure of the creation of individual assemblies and their opposing arrangement, etc. For the transition from the basic principle to its industrial use, however, it is necessary to find a viable procedure for large-size equipment.

It is evident that a device based on this principle makes it possible to achieve an even stronger magnetic field in a comparable air gap than presented in this paper - for example by the use of stronger magnets with a higher energy product, magnetic blocks of larger sizes, by the mentioned removal of stainless frames of the central magnetic blocks, the optimization of the dimensional proportions of Halbach arrays etc. Another promising possibility of achieving high magnetic induction in a large volume could be a parallel placement of two or more of these assemblies immediately next to each other. Of course, the essential prerequisite for the feasibility of this solution is its verification on a model device, mainly concerning the size achieved and the distribution of magnetic induction.

\section{Acknowledgments}

This work was carried out thanks to the support of the Long-term project for the conceptual development of the research organization No. RVO 67985891.

\section{References}

[1] Z. Wang, W. H. Yang, X. B. Zhang, L. L. Hu, and H. X. Wang, IEEE Trans. Appl. Supercond. 20, 3 (2010).

[2] F. Bloch, O. Cugat, G. Meunier, and J. C. Toussaint, IEEE Trans. Magn. 34, 5 (1998).

[3] Ch. Li and M. Devine, IEEE Trans. Magn. 41, 10 (2005).

[4] R. Bjørk, C. R. H. Bahl, A. Smith, and N. Pryds, IEEE Trans. Magn. 47, 6 (2011).

[5] V. Žežulka and P. Straka, IEEE Trans. Magn. 44, 4 (2008).

[6] N. Dogan, R. Topkaya, H. Subasi, Y. Yerli, and B. Rameev, J. of Physics D, Conference Series 153, 1 (2009). 
[7] V. C. Barroso, H. Raich, P. Blümler, and M. Wilhelm, J. of Physics D, Conference Series 149, 1 (2009).

[8] J. E. Hilton and S. M. McMurry, J. Magn. Magn. Mater. 324, 2051 (2012).

[9] A. Sarwar, A. Nemirovski, and B. Shapiro, J. Magn. Magn. Mater. 324, 742 (2012).

[10] J. Choi and J. Yoo, IEEE Trans. Magn. 44, 10 (2008).

[11] R. Bjørk, C. R. H. Bahl, A. Smith, and N. Pryds, J.
Magn. Magn. Mater. 322, 3664 (2010).

[12] A. E. Marble, IEEE Trans. Magn. 44, 5 (2008).

[13] V. Žežulka, J. Pištora, M. Lesňák, P. Straka, D. Ciprian, and J. Foukal, J. Magn. Magn. Mater. 345, 7 (2013).

[14] J. Jin, The Finite Element Method in Electromagnetics, J. Wiley \& Sons Inc., New York (2002).

[15] V. Žežulka, P. Straka, and V. Soukup, US Patent No. US 7,796,001 (2010). 